\title{
Screening of Most Effective Variables for Development of Gastroretentive Mucoadhesive Nanoparticles by Taguchi Design
}

\author{
Ankit Anand Kharia ${ }^{1,2}$ and Akhlesh Kumar Singhai ${ }^{2,3}$ \\ ${ }^{1}$ Department of Pharmaceutics, Oriental College of Pharmacy, Thakral Nagar, Raisen Road, Bhopal, Madhya Pradesh 462021, India \\ ${ }^{2}$ Department of Pharmacy, Uttarakhand Technical University, Sudhowala, Dehradun, Uttarakhand 248007, India \\ ${ }^{3}$ Department of Pharmaceutics, Lakshmi Narain College of Pharmacy, Raisen Road, Bhopal, Madhya Pradesh 462021, India
}

Correspondence should be addressed to Ankit Anand Kharia; ankitanandkharia@yahoo.co.in

Received 13 June 2013; Accepted 6 July 2013

Academic Editors: Z. Jiang, Z.-M. Liao, J. Shen, and W. W. Shu

Copyright (C) 2013 A. A. Kharia and A. K. Singhai. This is an open access article distributed under the Creative Commons Attribution License, which permits unrestricted use, distribution, and reproduction in any medium, provided the original work is properly cited.

\begin{abstract}
The objective of this study was the selection of the most influential variable for the preparation of gastroretentive mucoadhesive nanoparticles of acyclovir. Nanoparticles were prepared by one-step desolvation method; effect of formulation and processing variables on various response variables were studied by a Taguchi standard orthogonal array L8 design. Independent variables studied were the amount of gelatin, amount of glutaraldehyde, amount of Pluronic F-68, acetone addition rate, $\mathrm{pH}$, stirring time, and stirring speed. The dependent variables studied were the particle size, polydispersity index, amount of drug released in $6 \mathrm{~h}$, time required to release $60 \%$ of drug, entrapment efficiency, loading efficiency, and mucoadhesiveness. The size of all nanoparticulate formulations prepared as per the experimental design (Taguchi screening design) varied between 165 and $1610 \mathrm{~nm}$, PDI between 0.360 and 1.00, bioadhesiveness between 3.959 and $11.02 \mathrm{~g}$, cumulative percent drug release in $24 \mathrm{~h}$ between 40.74 and 72.48 , entrapment efficiency between 15.70 and 83.12, and loading efficiency between 39.72 and 80.49. Pareto ranking analyses showed that the two most important factors affecting the selected responses were amount of gelatin and amount of Pluronic F-68 $(P<0.05)$.
\end{abstract}

\section{Introduction}

Acyclovir is a cyclic analogue of the natural nucleoside 2deoxyguanosine, clinically used in the treatment of Herpes simplex, Varicella zoster, Cytomegalovirus, and Epstein barr virus infections [1]. It is currently marketed as capsules (200 mg), tablets (200, 400 and $800 \mathrm{mg}$ ), and suspension for oral administration, topical ointment, and intravenous injection. Oral acyclovir is mostly used as $200 \mathrm{mg}$ tablets, five times a day [2]. Absorption of orally administered acyclovir is slow, variable, and incomplete, with a bioavailability of $15 \%-30 \%$ and the elimination half-life of acyclovir is approximately $3 \mathrm{~h}$ [3]. It has narrow absorption window and is primarily absorbed from stomach and upper part of the small intestine [4-6]. Reduced bioavailability of acyclovir may be because of transportation of dosage form from the region of absorption window to site where it is less absorbed. Therefore there was a need to increase the gastroretention time of dosage form so that drug would be available at the site of absorption and results in improved bioavailability.

Several attempts are being made to increase the gastric retention of drugs, like intragastric floating systems, hydrodynamically balanced systems, extendable or expandable, microporous compartment system, microballoons, bio/mucoadhesive systems, high-density systems, and superporous biodegradable hydro gel systems [7]. After oral administration, such a dosage form would be retained in the stomach for several hours and would release the drug there in a controlled and prolonged manner, so that the drug could be supplied continuously to its absorption sites in the upper gastrointestinal tract [8]. Prolonged gastric retention improves bioavailability, reduces drug waste, and improves solubility of drugs that are less soluble in a high $\mathrm{pH}$ environment. It is also suitable for local drug delivery to the stomach and proximal small intestine [9]. Gastroretention helps to provide better 
availability of new products with suitable therapeutic activity and substantial benefits for patients.

The traditional approach of optimizing a formulation or process involves studying the influence of one variable at time (OVAT), while keeping others as constant. Using this OVAT approach, the solution of a specific problematic property can be achieved somehow, but attainment of the true optimum composition or process is never assured [10]. This may be due to presence of interactions, that is, the influence of one or more factors on others. The ultimate product may be satisfactory but mostly suboptimal, as a better formulation might still exist for the studied conditions. The conventional OVAT approach of drug formulation development suffers from several pitfalls, like being strenuous, uneconomical, and inept to reveal interactions. Further, the OVAT methodology results only in "just satisfactory" solutions, as a detailed study of all variables is not possible. As one cannot establish "causeand-effect" relationships using OVAT, it becomes ineffective when all variables are changed simultaneously. Recently, the systematic optimization approaches are being widely practiced to alleviate such inconsistencies. This holistic approach encompassing the application of appropriate experimental designs coupled with the generation of mathematical equations and graphic outcomes and depicting a complete picture of variation of the response(s) as a function of the factor(s) is termed as design of experiments (DoE). DoE techniques are thus far more beneficial, as they overcome most shortcomings inherent to the traditional OVAT approach [11, 12].

Further, the screening techniques employed as a part of DoE help in finding the "important" and "unimportant" input variables. One can simulate the product or process behavior using model equation(s) and thus save a significant amount of resources, namely, time, effort, materials, and cost. The remarkable feature of $\mathrm{DoE}$ is that it can predict the performance of formulations even without preparing them and detect and estimate the possible interactions and synergies among variables. Recently, DoE optimization techniques are becoming a regular practice globally, not only in the design and development of an assortment of new dosage forms but also for modifying the existing ones. Whether it is a drug industry, institutional drug delivery resource, or federal compliance with USFDA, ICH, NIH, or ISO, DoE is being frequently sought after in drug discovery and development. The faster emerging area of quality by design $(\mathrm{QbD})$ also requires the implementation of DoE precepts during different stages of product/process transformation. By and large, low-resolution designs like FDs (full or fractional), Plackett Burman design, or Taguchi designs are enough for the purpose of simpler screening of a large number of experimental parameters [10].

Taguchi methods or an orthogonal array have been widely used to optimize the reaction variables by formulating a minimum number of experiments. This approach helps to identify the influence of individual factors and establish the relationship between variables and operational conditions [13]. It is a method of designing experiments that usually requires only a fraction of the full factorial combinations. An orthogonal array means the design is balanced so that factor levels are weighted equally. Because of this, each factor can be evaluated independently of all the other factors, so the effect of one factor does not influence the estimation of another factor.

The aim of the present study was to screen most influential factors which effect formulation of mucoadhesive gastroretentive nanoparticles of acyclovir by Taguchi approach. Gelatin was selected as a mucoadhesive polymer to prepare gastroretentive nanoparticles as they strengthen the contact between dosage form and the site of absorption, thereby reducing the luminal diffusion pathway of the drug and lead to significant improvements in oral drug delivery [14, 15]. These mucoadhesive polymeric nanoparticles in the stomach will offer diverse advantages such as (a) longer residence time of the dosage form on gastric mucosa which will improve absorption of the drug and increase the bioavailability, (b) higher drug concentration at the site of adhesion absorption, which will create a driving force for the paracellular passive uptake, and (c) immediate absorption from the bioadhesive drug delivery system without previous dilution and probable degradation in the luminal fluids [16].

\section{Materials and Methods}

2.1. Materials. Acyclovir was obtained as a gift sample from M/s Modern Laboratories Pvt., Ltd., Indore, India. Dialysis tubing (cut-off $12 \mathrm{kDa}$ ) was purchased from Sigma (USA). Gelatin (type B) and Pluronic F-68 were purchased from HiMedia Laboratories Pvt., Ltd., Mumbai, India. All other ingredients used throughout the study were of analytical grade and were used as received.

2.2. Preparation of Nanoparticles. Gelatin nanoparticles were prepared by desolvation method as previously described [17] with slight modification. Briefly gelatin was dissolved in $25 \mathrm{~mL}$ of distilled water at $37^{\circ} \mathrm{C}$ under magnetic stirring (400 rpm) until a clear solution was obtained; $200 \mathrm{mg}$ acyclovir was dispersed in $50 \mathrm{~mL}$ of distilled water. Drug dispersion was then added to the polymeric solution followed by addition of Pluronic F-68 as a stabilizer under continuous stirring by using magnetic stirrer and the $\mathrm{pH}$ of solution was adjusted (by $1 \mathrm{~mol} / \mathrm{L} \mathrm{HCl}$ or $\mathrm{NaOH}$ ). Then $50 \mathrm{~mL}$ of acetone was added at specified addition rate, after $10 \mathrm{~min}$ of acetone addition glutaraldehyde was added to the reaction vessel to cross-link the nanoparticles. Finally after stirring for $6 \mathrm{~h}$, the particles were purified by threefold centrifugation $(16000 \mathrm{~g}$ for $20 \mathrm{~min}$ at $4^{\circ} \mathrm{C}$ ) and redispersion in $10 \mathrm{~mL}$ mixture of acetone: water $(3: 7 \mathrm{v} / \mathrm{v})$. The supernatant was removed and the pellets were resuspended in distilled water, and finally, the nanoparticles were freeze-dried using $5 \%$ glucose solution as a cryoprotector and powder was stored in vials.

\subsection{Evaluation of Formulations}

2.3.1. Particle Size and Particle Size Distribution. Average particle size and polydispersity index (PDI) which is a range of measurement of the particle sizes within measured samples were determined using the zeta sizer (Zetasizer-ZEN 2600 Malvern Instrument, Ltd., Worchestershire, UK) equipped with the Malvern PCS software. For analysis nanosuspensions were diluted five times with filtered $(0.45 \mu)$ bidistilled water [18]. 
2.3.2. Entrapment Efficiency. For determination of drug entrapment, the amount of drug present in the clear supernatant after centrifugation was determined $(w)$ by UV spectrophotometer at $254 \mathrm{~nm}$ (UV-1700 Spectrophotometer, Shimadzu Scientific Instruments, Inc., Maryland, USA). A standard calibration curve of drug was plotted for this purpose. The amount of drug in supernatant was then subtracted from the total amount of drug added during the preparation $(W)$. Effectively, $(W-w)$ will give the amount of drug entrapped in the particles [19].

Then percentage entrapment of a drug is obtained by using the following equation:

$$
\% \text { Drug Entrapment }=(W-w) \times \frac{100}{W} .
$$

2.3.3. Drug Loading. The acyclovir content in the nanoparticles was determined by pulverizing the acyclovir loaded nanoparticles $(10 \mathrm{mg}$ ) followed by immersing them in $100 \mathrm{~mL}$ simulated gastric fluid (SGF, pH 1.2, without enzymes) with agitating at room temperature for $12 \mathrm{~h}$. After filtration through a $0.45 \mu$ membrane filter (Millipore), the drug concentration was determined spectrophotometrically at the wavelength of $254 \mathrm{~nm}$. The filtered solution from the empty nanoparticles (without acyclovir) was taken as blank. All samples were analyzed in triplicate and the drug loading (DL) was calculated according to the following equation [20]:

$$
\mathrm{DL}(\%)=\frac{\mathrm{WD}}{\mathrm{WT}} \times 100 \text {, }
$$

where DL is the drug loading; WD is the weight of the drug loaded in the nanoparticles; WT is the total weight of the nanoparticles.

2.3.4. Drug Release Study. The in vitro drug release studies were performed by dialysis membrane diffusion technique using glass tube of $10 \mathrm{~cm}$ length open at its both ends having $2.5 \mathrm{~cm}$ diameter. The dialysis membrane of 12,000 Mwco (Spectra por, Sigma, USA) was used for release study because it retains nanoparticles and allows free drug to diffuse in the release media. The lower end of the glass tube was covered with the pretreated membrane to keep the nanoparticulate formulation on the donor side. The nanoparticles (equivalent to $10 \mathrm{mg}$ of acyclovir) were placed in donor compartment by dispersing in $3 \mathrm{~mL}$ of SGF ( $\mathrm{pH}$ 1.2) where the drug was allowed to freely diffuse over the receptor compartment containing $100 \mathrm{~mL}$ of SGF (pH 1.2). The entire system was kept at $37 \pm 0.5^{\circ} \mathrm{C}$ with continuous magnetic stirring at $100 \mathrm{rpm}$. Samples of $5 \mathrm{~mL}$ were withdrawn at predetermined time intervals $(0.5,1,2,3,4,5,6,12$, and $24 \mathrm{~h})$ and replaced with fresh SGF $[18,21]$. The withdrawn samples were suitably diluted to carry out UV Spectrophotometric analysis at $254 \mathrm{~nm}$.

2.3.5. Measurement of Mucoadhesive Strength. The method is based on the measurement of shear stress required to break the adhesive bond between a mucosal membrane and the formulation. The formulation is sandwiched between
TABLE 1: List of variables employed in Taguchi L8 orthogonal array experiment.

\begin{tabular}{lcc}
\hline \multirow{2}{*}{ Factors } & \multicolumn{2}{c}{ Levels } \\
& Low $(-1)$ & High $(+1)$ \\
\hline Amount of gelatin $(\mathrm{mg})$ & 200 & 1000 \\
Amount of glutaraldehyde $(\mathrm{mL})$ & 0.1 & 0.5 \\
Amount of pluronic F-68 $(\mathrm{mg})$ & 50 & 250 \\
Acetone addition rate $(\mathrm{mL} / \mathrm{min})$ & 1 & 5 \\
pH & 2 & 4 \\
Stirring time $(\mathrm{h})$ & 1 & 5 \\
Stirring speed $(\mathrm{rpm})$ & 200 & 1000 \\
\hline
\end{tabular}

two mucosal membranes fixed on flexible supports in the assemblies for a sufficient period of time. After the adhesive bond has formed, the force (weight) required to separate the bond was recorded as mucoadhesive strength.

Mucoadhesive properties of acyclovir nanoparticles were evaluated by Texture analyzer (M/s TA. XT. Plus, Stable Microsystem, UK) using porcine gastric mucosa. Stomach of pig was obtained immediately after slaughter at local slaughterhouse. The stomach was washed with fresh water to remove nondigested food from stomach, then placed in SGF at $4^{\circ} \mathrm{C}$ (used within $6 \mathrm{~h}$ ). The membrane was then attached both on the base of texture analyzer and to the stainless steel probe (using two-side adhesive tape); probe is then fixed to the mobile arm of the texture analyzer. The $10 \mathrm{mg}$ of nanoparticulate formulation was placed on the membrane placed on lower surface moistened with $1 \mathrm{~mL}$ of SGF. The mobile arm (with attached membrane) was lowered at a rate of $0.5 \mathrm{~mm} \mathrm{~s}^{-1}$ until contact with the formulation was made. A contact force of $10 \mathrm{~g}$ was maintained for $500 \mathrm{~s}$, after which the probe was withdrawn from the membrane. After the adhesive bond has formed, the force of detachment $(\mathrm{g})$ required to separate the bond was recorded as mucoadhesive strength [22]. This parameter was used to compare mucoadhesive property of various formulations.

2.4. Screening of Influential Variables. A number of formulation and processing variables influence the overall performance of nanoparticles. Thus it becomes extremely difficult to study the effect of each variable and interaction among them through the conventional approach. In current studies, screening of various process and formulation variables, potentially influencing nanoparticulate formulation development, was performed employing Taguchi design for seven factors at two levels each as given in Table 1 . The number of experiments during screening was kept as small as possible, to limit the volume of work carried out during initial stages. This was conducted to identify the potential factors for further systematic DoE optimization studies, as the nature of influence of such variables is not widely known from prior literature reports.

A standard orthogonal array L8 was used to examine this system where $\mathrm{L}$ and subscript 8 denote the latin square and the number of the experimental runs, respectively. Design-Expert (Stat-Ease Inc., USA) was used as software for 
TABLE 2: L8 array layout as per 7 factors, 2 levels Taguchi screening design.

\begin{tabular}{|c|c|c|c|c|c|c|c|}
\hline $\begin{array}{l}\text { Formulation } \\
\text { code }\end{array}$ & $\begin{array}{l}\text { Amount of } \\
\text { gelatin (mg) }\end{array}$ & $\begin{array}{c}\text { Amount of } \\
\text { Glutaraldehyde } \\
(\mathrm{mL})\end{array}$ & $\begin{array}{c}\text { Amount of } \\
\text { Pluronic F-68 } \\
(\mathrm{mg})\end{array}$ & $\begin{array}{c}\text { Acetone } \\
\text { addition rate } \\
(\mathrm{mL} / \mathrm{min})\end{array}$ & $\mathrm{pH}$ & Stirring time $(\mathrm{h})$ & $\begin{array}{l}\text { Stirring speed } \\
\quad(\mathrm{rpm})\end{array}$ \\
\hline $\mathrm{F} 1$ & -1 & -1 & -1 & -1 & -1 & -1 & -1 \\
\hline $\mathrm{F} 2$ & -1 & +1 & -1 & +1 & +1 & +1 & -1 \\
\hline F3 & +1 & +1 & -1 & -1 & -1 & +1 & +1 \\
\hline $\mathrm{F} 4$ & +1 & -1 & -1 & +1 & +1 & -1 & +1 \\
\hline F5 & +1 & -1 & +1 & +1 & -1 & +1 & -1 \\
\hline F6 & +1 & +1 & +1 & -1 & +1 & -1 & -1 \\
\hline F7 & -1 & +1 & +1 & +1 & -1 & -1 & +1 \\
\hline $\mathrm{F} 8$ & -1 & -1 & +1 & -1 & +1 & +1 & +1 \\
\hline
\end{tabular}

TABLE 3: Preparation of nanoparticles on the basis of Taguchi screening design.

\begin{tabular}{|c|c|c|c|c|c|c|c|}
\hline Formulation & $\begin{array}{c}\text { Amount of } \\
\text { gelatin (mg) }\end{array}$ & $\begin{array}{c}\text { Amount of } \\
\text { Glutaraldehyde } \\
(\mathrm{mL})\end{array}$ & $\begin{array}{c}\text { Amount of } \\
\text { Pluronic F-68 } \\
\text { (mg) }\end{array}$ & $\begin{array}{c}\text { Acetone } \\
\text { addition rate } \\
(\mathrm{mL} / \mathrm{min})\end{array}$ & $\mathrm{pH}$ & Stirring time $(\mathrm{h})$ & $\begin{array}{l}\text { Stirring speed } \\
(\mathrm{rpm})\end{array}$ \\
\hline F1 & 200 & 0.1 & 50 & 1 & 2.0 & 1 & 200 \\
\hline $\mathrm{F} 2$ & 200 & 0.5 & 50 & 5 & 4.0 & 5 & 200 \\
\hline F3 & 1000 & 0.5 & 50 & 1 & 2.0 & 5 & 1000 \\
\hline $\mathrm{F} 4$ & 1000 & 0.1 & 50 & 5 & 4.0 & 1 & 1000 \\
\hline F5 & 1000 & 0.1 & 250 & 5 & 2.0 & 5 & 200 \\
\hline F6 & 1000 & 0.5 & 250 & 1 & 4.0 & 1 & 200 \\
\hline F7 & 200 & 0.5 & 250 & 5 & 2.0 & 1 & 1000 \\
\hline $\mathrm{F} 8$ & 200 & 0.1 & 250 & 1 & 4.0 & 5 & 1000 \\
\hline
\end{tabular}

Drug amount was kept constant at $200 \mathrm{mg}$.

the DoE modeling. Table 2 depicts the orthogonal array L8 for the seven factors at two levels by Taguchi design was adopted in the studies. Particle size, polydispersity index (PDI), amount of drug released in $6 \mathrm{~h}\left(Q_{6}\right)$, time required to release $60 \%$ of drug $\left(T_{60 \%}\right)$, entrapment efficiency, loading efficiency, and mucoadhesiveness were the key response variables investigated thoroughly for selecting the significant formulation and response factors. A run involved the corresponding combination of levels to which the factors in the experiment were set as shown in Table 3. All experiments were done in triplicate. The effects of the proposed experiments on the responses were then analyzed by the Design Expert software to obtain independently the main effects of these factors, followed by the analysis of variance (ANOVA) to determine which factors were statistically significant. Series of Pareto charts were constructed to demonstrate the influence of each parameter on the responses (Figure 3). The most influential factor among the seven factors will be derived by selecting factors which are showing maximum standardized effect for most of the responses in Pareto charts.

\section{Results and Discussion}

The current study was undertaken to screen most influential factors in optimization of a nanoparticulate system suitable for mucoadhesion along with low particle size, low PDI, sustained release, high entrapment, and loading efficiency. The results for evaluation of gastroretentive mucoadhesive nanoparticles are given in Table 4 .

\subsection{Evaluation of Formulations}

3.1.1. Influence of Various Factors on Particle Size. It has been shown that particle size and size distribution are the most important characteristics of nanoparticles systems. Many studies have demonstrated that nanoparticles of submicron size have a number of advantages over microparticles as a drug delivery system. It was found that increasing the quantity of Pluronic F-68 results in reduction in size of the formulation because it decreases the surface tension between organic and aqueous phase and leads to the formation of smaller solvent droplets, which in turn causes decrease in particle size. It also stabilizes newly generated surfaces and prevents aggregation of the particles which is similar to the previous studies [23]. It was also observed that increase in stirring speed and amount of cross-linking agent results in reduction in particle size which may be due to stabilization of the surface and thereby preventing aggregation in later case. Lower value of $\mathrm{pH}$ favors nanoparticles with small size because of maximum solubility of gelatin at $\mathrm{pH}$ 
TABLE 4: Evaluation of formulations of Taguchi screening design.

\begin{tabular}{lccccccc}
\hline Formulation & $\begin{array}{c}\text { Particle Size } \\
(\mathrm{nm})\end{array}$ & PDI & $\mathrm{Q}_{6}{ }^{*}(\%)$ & $\mathrm{T}_{60 \%}{ }^{*}(\mathrm{~h})$ & $\begin{array}{c}\text { Entrapment } \\
\text { efficiency }{ }^{*}(\%)\end{array}$ & $\begin{array}{c}\text { Drug loading* } \\
(\%)\end{array}$ & $\begin{array}{c}\text { Mucoadhesion } \\
\text { study }{ }^{*}(\mathrm{~g})\end{array}$ \\
\hline F1 & 165.3 & 0.906 & $15.00 \pm 0.26$ & $26.8 \pm 0.351$ & $20.26 \pm 1.98$ & $39.72 \pm 1.03$ & $6.021 \pm 0.094$ \\
F2 & 256.7 & 1.000 & $14.34 \pm 0.74$ & $24.4 \pm 0.861$ & $30.82 \pm 1.45$ & $53.04 \pm 1.14$ & $4.494 \pm 0.058$ \\
F3 & 478.3 & 0.832 & $34.93 \pm 0.86$ & $21.2 \pm 0.984$ & $15.70 \pm 2.16$ & $47.25 \pm 2.01$ & $11.02 \pm 0.846$ \\
F4 & 1610 & 0.360 & $7.31 \pm 0.64$ & $37.6 \pm 0.654$ & $51.69 \pm 2.48$ & $55.07 \pm 2.48$ & $8.347 \pm 0.989$ \\
F5 & 552.8 & 0.532 & $12.77 \pm 0.26$ & $21.2 \pm 1.48$ & $31.57 \pm 1.61$ & $78.63 \pm 1.98$ & $5.992 \pm 0.114$ \\
F6 & 345.9 & 1.000 & $7.59 \pm 1.29$ & $37.2 \pm 2.03$ & $49.30 \pm 3.04$ & $80.49 \pm 2.85$ & $5.029 \pm 0.0742$ \\
F7 & 319.3 & 0.656 & $11.89 \pm 2.35$ & $24 \pm 0.987$ & $83.12 \pm 2.45$ & $59.04 \pm 1.64$ & $3.959 \pm 0.056$ \\
F8 & 1300 & 0.809 & $15.42 \pm 0.35$ & $19.2 \pm 0.494$ & $67.79 \pm 1.47$ & $71.23 \pm 1.03$ & $5.029 \pm 0.081$ \\
\hline
\end{tabular}

${ }^{*}$ Mean \pm SD $(n=3) ;(P<0.05)$.

$Q_{6}$ is amount of drug released in $6 \mathrm{~h} ; T_{60 \%}{ }^{*}(\mathrm{~h})$ is time required for $60 \%$ cumulative drug release.

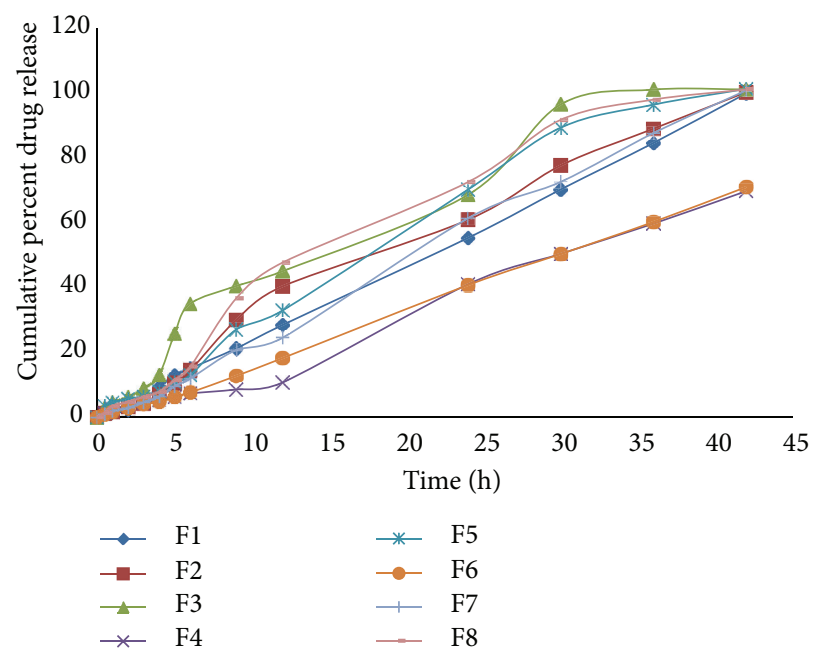

FIGURE 1: Drug release study of formulations of Taguchi screening design.

values far from its isoelectric point $(\mathrm{pH} 4.7-5.2$ for gelatin type B).

3.1.2. Influence of Various Factors on Particle Size Distribution. It was found that on increasing $\mathrm{pH}$ (close to isoelectric point of gelatin) and amount of glutaraldehyde PDI was increased, while high stirring speed and amount of polymer resulted in lowering of PDI. Considering that the PDI is calculated from the square of the standard deviation/mean diameter, less value of polydispersity index indicates enhanced homogeneity of the nanoparticles.

3.1.3. Influence of Various Factors on Loading and Entrapment Efficiency. Loading efficiency of the formulation increases with increasing the amount of polymer. Maximum loading and entrapment efficiency was obtained when drug is loaded at or near its isoelectric point when it has minimum solubility and maximum adsorption. Loading and entrapment efficiencies were more on increasing the acetone addition rate and
Pluronic F-68. On increasing stirring speed loading efficiency reduces which may be because of leaching of drug from the formulation before cross-linking step. It is probable that the entrapment of acyclovir molecule to gelatin nanoparticles is due to noncovalent interactions since gelatin (type $\mathrm{B}$, isoelectric point between $\mathrm{pH} 4.7-5.2$ ) remains charged at a $\mathrm{pH}$ away from isoelectric point. Noncovalent interactions unlike covalent types proceed more slowly and go to completion over time results in increased loading efficiency with increase in stirring time.

3.1.4. Influence of Various Factors on Drug Release. The results of drug release study for various formulations are shown in Figure 1. Drug release was found to decrease with increase in the polymer ratio. This can be attributed to the release retarding effect of polymer which reduces the release of drug from the nanoparticulate formulation. Release rate was also reduced with increase in amount of cross-linking agent which may be due to rigidization of surface of nanoparticles. 


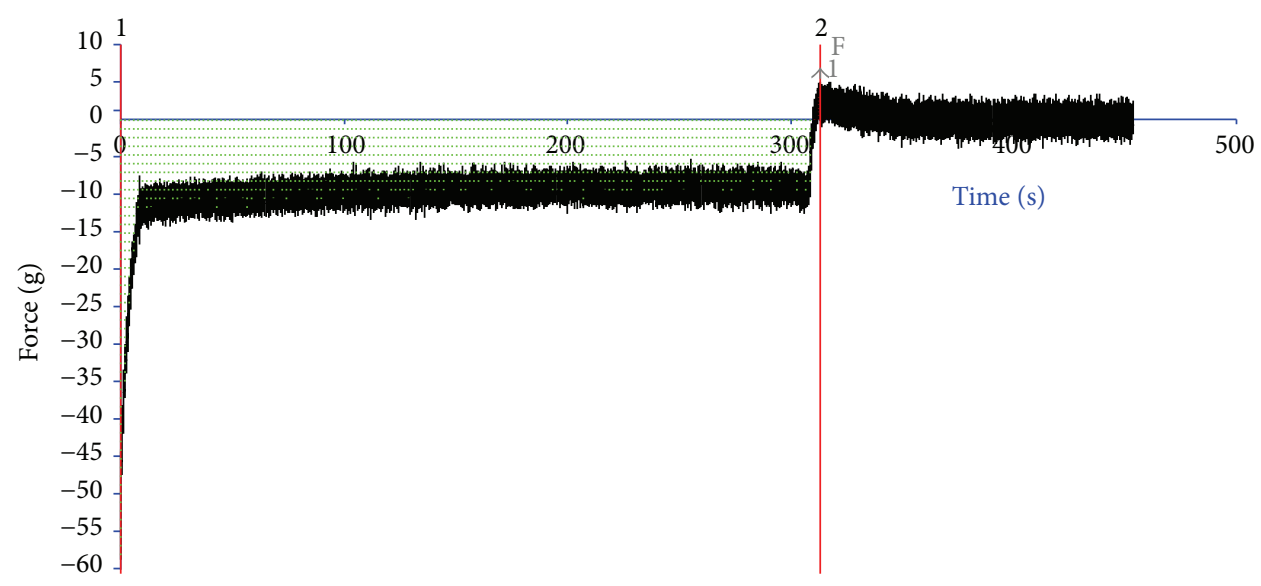

FIGURE 2: Graph showing force of detachment. Point F represents the force at which the breaking of adhesive bond starts (the force of detachment).

3.1.5. Influence of Various Factors on Mucoadhesion. Mucoadhesion studies were performed by using texture analyzer and representative graph showing force of detachment is given in Figure 2. Point $F$ represents the force at which the breaking of the adhesive bond starts (i.e., the force of detachment). It was found that increase in polymer ratio increases the mucoadhesiveness of the formulation due to bioadhesive nature of the polymer, while increase in concentration of Pluronic F-68 and glutaraldehyde results in lowering of mucoadhesiveness which may be due to surfactant effect of Pluronic F-68 and greater cross-linking of mucoadhesive polymer due to glutaraldehyde.

3.2. Screening of Influential Formulation and Process Variables. Taguchi has developed a method based on "orthogonal array" experiments which gives much reduced "variance" for the experiment with "optimum settings" of control parameters. Seven formulation and process variables including amount of gelatin $(\mathrm{mg})$, amount of glutaraldehyde $(\mathrm{mL})$, amount of Pluronic F-68 (mg), acetone addition rate $(\mathrm{mL} / \mathrm{min}), \mathrm{pH}$, stirring time $(\mathrm{h})$, and stirring speed were studied each in two levels.

Implementation of the L8 array design helps in identifying the most significant factors for further detailed investigation with minimum experimentation thus saving considerable time, efforts, and resources. Data obtained after the evaluation of the prepared formulation was subjected to ANOVA by using Design Expert Software which provides the coefficient values for the selected responses. These coefficient values were used as standardized effect and plotted against various responses to construct Pareto charts. The "standardized effect" determines each factor's relative strength; the higher the absolute value the greater the effect of that factor on the response. A positive effect value indicates an effect that favors the response, and a negative value represents an inverse relationship between the response and the factor. Pareto ranking analyses showed that the most significant factors were amount of gelatin and amount of Pluronic F-68 $(P<0.05)$ relative to other factors influencing the studied response variables including particle size, $\mathrm{PDI}, Q_{6}, T_{60 \%}$, entrapment efficiency, loading efficiency, and mucoadhesiveness.

Based on the results of Taguchi screening further experimental design such as central composite design is suggested to generate polynomial equations for various responses, feasibility followed by intensive grid search, and overlay plot method for selection of optimized formulation. The criteria for selection of suitable feasible region will be based upon the best possible values of particle size, PDI, $Q_{6}, T_{60 \%}$, entrapment efficiency, loading efficiency, and mucoadhesiveness. Validated formulations will be evaluated for their performance and the results will be critically compared with those predicted using RSM.

\section{Conclusion}

A Taguchi design was performed to screen the effect of formulation and processing variables on the response variables by applying computer optimization technique. Screening studies showed that the most influential independent variables were amount of gelatin and amount of Pluronic F-68 which influences the studied response variables for development of gastroretentive mucoadhesive nanoparticles. Based on the results of optimization studies it was concluded that further experimental designs are required to study the main interaction, quadratic effects of these variables on the responses and obtain the optimized formulation.

\section{Conflict of Interests}

The authors report no declarations of interest.

\section{Acknowledgments}

The authors are thankful to Modern Laboratories (Indore, India) for providing gift sample of drug and SAIL, RGPV, 

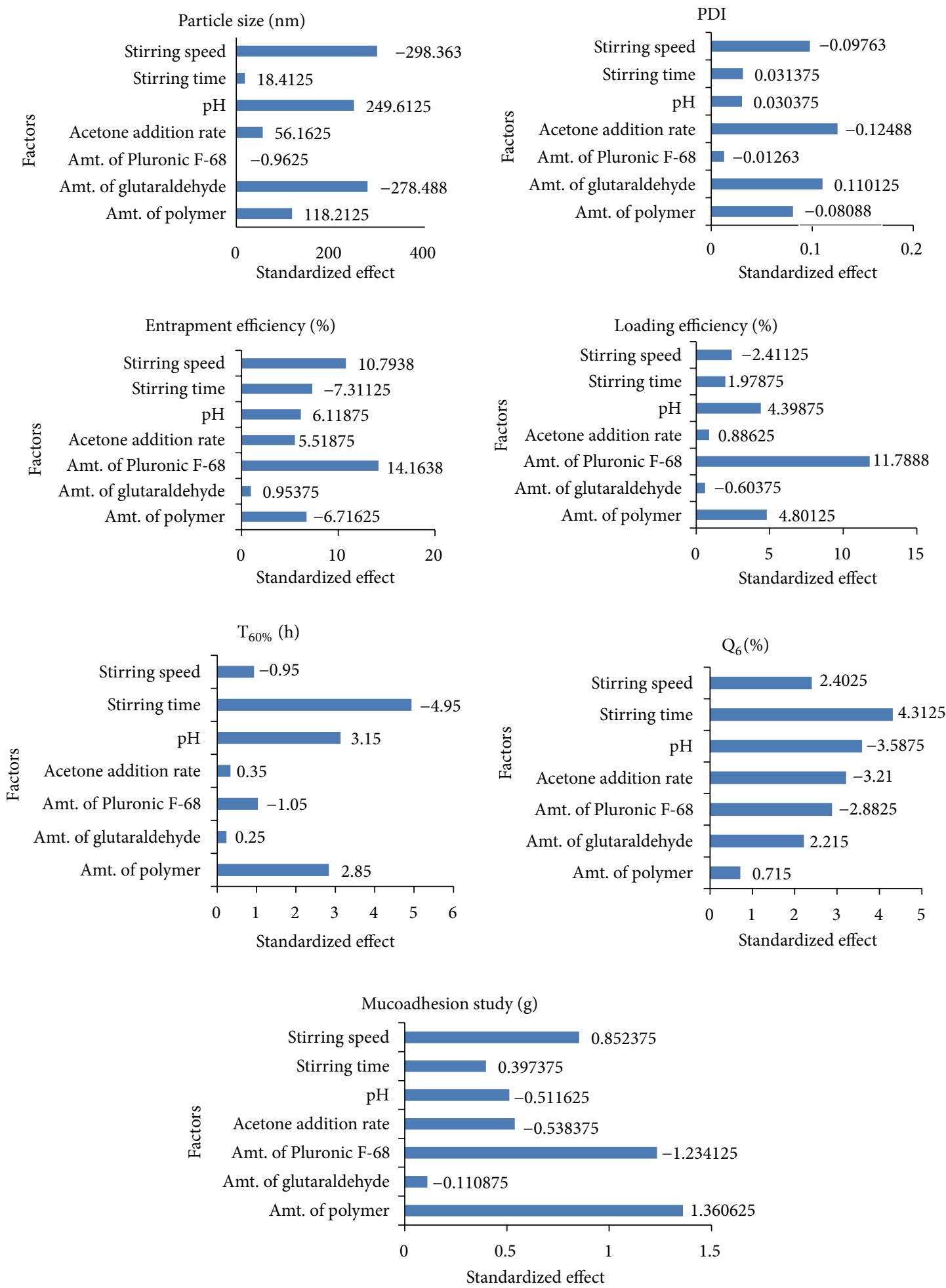

FIGURE 3: Pareto chart of the standardized effects of various formulation and process factors on selected responses. 
Bhopal, India, for their valuable contribution in the determination of particle size and PDI of the formulations. The authors are also thankful to UIPS, CAS, Panjab University, Chandigarh, India for texture analysis.

\section{References}

[1] J. J. O’Brien and D. M. Campoli-Richards, "Acyclovir. An updated review of its antiviral activity, pharmacokinetic properties and therapeutic efficacy," Drugs, vol. 37, no. 3, pp. 233-309, 1989.

[2] M. Ruhneck-Forsbeck, E. Sandstrom, and B. Andersson, "Treatment of recurrent genital herpes simplex infections with oral acyclovir," Journal of Antimicrobial Chemotherapy, vol. 16, no. 5, pp. 621-628, 1985.

[3] Z. Shao and A. K. Mitra, "Bile salt-fatty acid mixed micelles as nasal absorption promoters. III. Effects of nasal transport and enzymatic degradation of acyclovir prodrugs," Pharmaceutical Research, vol. 11, no. 2, pp. 243-250, 1994.

[4] R. Gröning, M. Berntgen, and M. Georgarakis, "Acyclovir serum concentrations following peroral administration of magnetic depot tablets and the influence of extracorporal magnets to control gastrointestinal transit," European Journal of Pharmaceutics and Biopharmaceutics, vol. 46, no. 3, pp. 285-291, 1998.

[5] K. C. Meadows and J. B. Dressman, "Mechanism of acyclovir uptake in rat jejunum," Pharmaceutical Research, vol. 7, no. 3, pp. 299-303, 1990.

[6] K. Park and J. R. Robinson, "Bioadhesive polymers as platforms for oral-controlled drug delivery: method to study bioadhesion," International Journal of Pharmaceutics, vol. 19, no. 2, pp. 107-127, 1984.

[7] B. N. Singh and K. H. Kim, "Floating drug delivery systems: an approach to oral controlled drug delivery via gastric retention," Journal of Controlled Release, vol. 63, no. 3, pp. 235-259, 2000.

[8] A. Streubel, J. Siepmann, and R. Bodmeier, "Gastroretentive drug delivery systems," Expert Opinion on Drug Delivery, vol. 3, no. 2, pp. 217-233, 2006.

[9] A. A. Kharia, S. N. Hiremath, A. K. Singhai, L. K. Omray, and S. K. Jain, "Design and optimization of floating drug delivery system of acyclovir," Indian Journal of Pharmaceutical Sciences, vol. 72, no. 5, pp. 599-606, 2010.

[10] B. Singh, R. Kumar, and N. Ahuja, "Optimizing drug delivery systems using systematic "design of experiments." Part I: fundamental aspects," Critical Reviews in Therapeutic Drug Carrier Systems, vol. 22, no. 1, pp. 27-105, 2005.

[11] B. Singh, S. K. Chakkal, and N. Ahuja, "Formulation and optimization of controlled release mucoadhesive tablets of atenolol using response surface methodology," AAPS PharmSciTech, vol. 7, no. 1, pp. E19-E28, 2006.

[12] B. Singh, S. Pahuja, R. Kapil, and N. Ahuja, "Formulation development of oral controlled release tablets of hydralazine: optimization of drug release and bioadhesive characteristics," Acta Pharmaceutica, vol. 59, no. 1, pp. 1-13, 2009.

[13] V. Venkata Dasu, T. Panda, and M. Chidambaram, "Determination of significant parameters for improved griseofulvin production in a batch bioreactor by Taguchi's method," Process Biochemistry, vol. 38, no. 6, pp. 877-880, 2003.

[14] H. L. Luessen, C.-M. Lehr, C.-O. Rentel et al., "Bioadhesive polymers for the peroral delivery of peptide drugs," Journal of Controlled Release, vol. 29, no. 3, pp. 329-338, 1994.
[15] G.-B. Park, Z. Shao, and A. K. Mitra, "Acyclovir permeation enhancement across intestinal and nasal mucosae by bile saltacylcarnitine mixed micelles," Pharmaceutical Research, vol. 9, no. 10, pp. 1262-1267, 1992.

[16] R. Hejazi and M. Amiji, "Chitosan-based gastrointestinal delivery systems," Journal of Controlled Release, vol. 89, no. 2, pp. 151165, 2003.

[17] C. J. Coester, K. Langer, H. Von Briesen, and J. Kreuter, “Gelatin nanoparticles by two step desolvation-a new preparation method, surface modifications and cell uptake," Journal of Microencapsulation, vol. 17, no. 2, pp. 187-193, 2000.

[18] A. H. Elshafeey, A. O. Kamel, and G. A. S. Awad, "Ammonium methacrylate units polymer content and their effect on acyclovir colloidal nanoparticles properties and bioavailability in human volunteers," Colloids and Surfaces B, vol. 75, no. 2, pp. 398-404, 2010.

[19] S. Das, R. Banerjee, and J. Bellare, "Aspirin loaded albumin nanoparticles by coacervation: implications in drug delivery," Trends in Biomaterials and Artificial Organs, vol. 18, no. 2, pp. 203-211, 2005.

[20] N. Ma, L. Xu, Q. Wang et al., "Development and evaluation of new sustained-release floating microspheres," International Journal of Pharmaceutics, vol. 358, no. 1-2, pp. 82-90, 2008.

[21] A. O. Kamel, G. A. S. Awad, A. S. Geneidi, and N. D. Mortada, "Preparation of intravenous stealthy acyclovir nanoparticles with increased mean residence time," AAPS PharmSciTech, vol. 10, no. 4, pp. 1427-1436, 2009.

[22] N. Thirawong, J. Nunthanid, S. Puttipipatkhachorn, and P. Sriamornsak, "Mucoadhesive properties of various pectins on gastrointestinal mucosa: an in vitro evaluation using texture analyzer," European Journal of Pharmaceutics and Biopharmaceutics, vol. 67, no. 1, pp. 132-140, 2007.

[23] M. A. Schubert and C. C. Müller-Goymann, "Solvent injection as a new approach for manufacturing lipid nanoparticlesevaluation of the method and process parameters," European Journal of Pharmaceutics and Biopharmaceutics, vol. 55, no. 1, pp. 125-131, 2003. 



Submit your manuscripts at http://www.hindawi.com
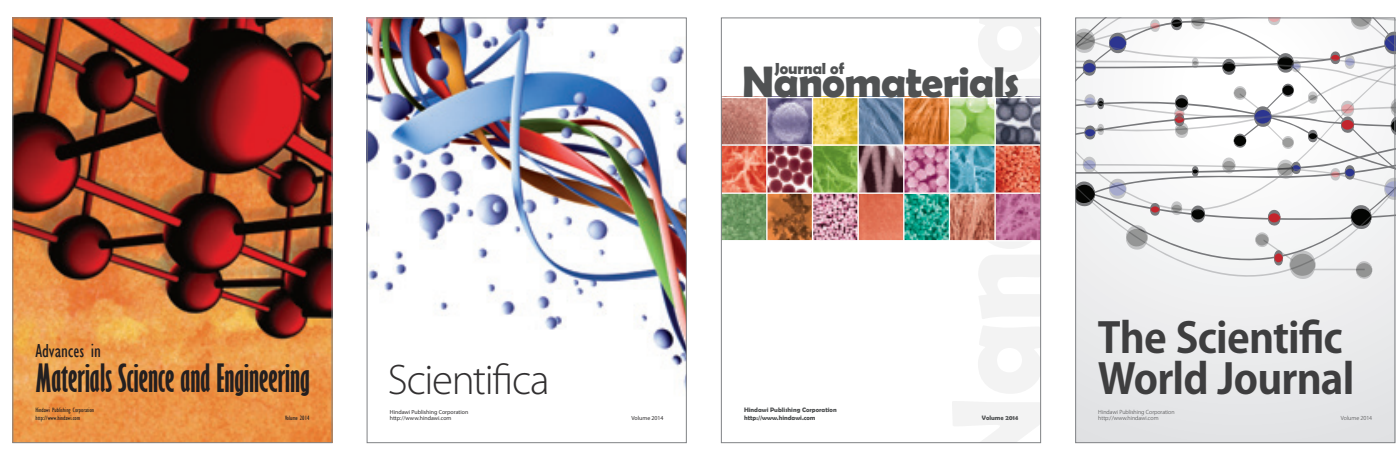

\section{The Scientific World Journal}
